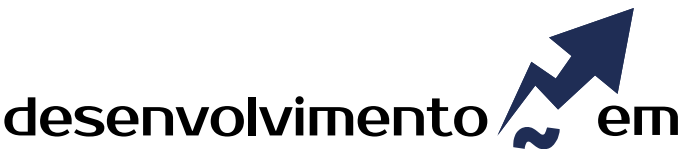 QUESTÃO
}

\section{Comparando Grupos de Agricultores de Acordo com suas Intenções em Diversificar a Produção Agrícola Uma Aplicação da Teoria do Comportamento Planejado}

\author{
http://dx.doi.org/10.21527/2237-6453.2019.48.353-371
}

Recebido em: 23/11/2018

Aceito em: 20/3/2019

\author{
Igor Senger ${ }^{1}$, João Augusto Rossi Borges², \\ João Armando Dessimon Machado ${ }^{3}$, Fernando Panno ${ }^{4}$
}

\begin{abstract}
RESUMO
A diversificação da produção no meio rural é um dos temas prioritários na União Europeia, uma vez que assegura a produção de diferentes alimentos. O objetivo deste estudo é analisar se os diferentes níveis de intenção dos agricultores familiares em diversificar a produção agrícola podem ser explicados pelos constructos Atitude, Norma Subjetiva e Controle Comportamental Percebido, pelos estilos de decisão e pelas características socioeconômicas dos agricultores. O estudo foi realizado com 101 agricultores familiares localizados na Região Noroeste do Estado do Rio Grande do Sul, Brasil, que tem na produção de leite sua principal fonte de renda. Os dados demonstraram que existem dois grupos de agricultores, um com fraca intenção de diversificar e outro com forte intenção. Constatou-se que o grupo com fraca intenção percebe a diversificação como menos favorável (atitude), sente uma menor pressão social (normas subjetivas) e percebe como menor sua capacidade de utilizar esta estratégia produtiva em seus estabelecimentos rurais (controle comportamental percebido) do que o grupo com forte intenção. Os resultados mostraram que o grupo com forte intenção de diversificar possui família maior e tem menor área de terra, comparado com o grupo de fraca intenção. Os resultados deste estudo podem ser utilizados para promover o desenvolvimento rural, e sugerem políticas públicas voltadas para este espaço, sobretudo para formas de produção que fornecem alimentos destinados ao abastecimento urbano.
\end{abstract}

Palavras-chave: Diversificação. Produção agrícola. Intenção. Teoria do comportamento planejado. Leite.

\section{COMPARING GROUPS OF FARMERS ACCORDING TO THEIR INTENTIONS TO DIVERSIFY} AGRICULTURAL PRODUCTION: AN APPLICATION OF THE PLANNED BEHAVIOR THEORY

\begin{abstract}
Diversification of production in rural areas is one of the priority themes in the European Union, since it ensures the production of different foods. The objective of this study is to analyze if the different levels of intention of the familiar farmers in diversifying the agricultural production can be explained by the constructs Attitude, Subjective Normative and Perceived Behavioral Control, by the decision styles and the socioeconomic characteristics of the farmers. The study was carried out with 101 family farmers located in the Northwest region of the state of Rio Grande do Sul, Brazil, where milk production is their main source of income. The data showed that there are two groups of farmers, one with weak intention to diversify and another with strong intention. It was observed that the group with low intention perceives diversification as less favorable (attitude), feel less social pressure (subjective norms) and perceive as less their ability to use this productive strategy in their rural establishments (perceived behavioral control) than the group with strong intention. The results showed that the group with a strong intention to diversify has a larger family and has a smaller area of land, compared to the group with low intention. The results of this study can be used to promote rural development in small farms and suggest public policies aimed at this area, especially for forms of production that provide food for urban supply.
\end{abstract}

Keywords: Diversification. Agricultural production. Intention. Theory of planned behavior. Milk.

\footnotetext{
${ }^{1}$ Doutor em Agronegócios pela Universidade Federal do Rio Grande do Sul (UFRGS). Professor da Universidade Federal de Santa Maria (UFSM), Frederico Westphalen. igorsenger@ufsm.br

2 Doutor em Business Economics pela Wageningen University, Professor da Universidade Federal da Grande Dourados (UFGD). joaoborges@ ufgd.edu.br

${ }^{3}$ Doutor em Economia Agroalimentar pela Universidade de Córdoba, Argentina. Professor da Universidade Federal do Rio Grande do Sul (UFRGS). joao.dessimon@ufrgs.br

${ }^{4}$ Doutor em Desenvolvimento Rural pela Universidade Federal do Rio Grande do Sul (UFRGS). Professor da Universidade Federal de Santa Maria (UFSM), Frederico Westphalen.fpanno@ufsm.br
} 
A diversificação da produção no meio rural situa-se ainda entre os temas prioritários na União Europeia quando se discutem políticas de desenvolvimento rural (HANSSON et al., 2013). Dentre os pontos discutidos, destaca-se a importância da diversificação das atividades agrícolas e não agrícolas para a geração de emprego e renda, diminuição da pobreza no meio rural, sua contribuição para o desenvolvimento rural e fortalecimento dos meios de vida dos indivíduos (ELLIS, 2000a; NORDER, 2009; SCHNEIDER, 2009). Pode ainda auxiliar no aumento da produção com uso de rendas não agrícolas, conservação do meio ambiente ante a necessidade de diminuir a exploração do solo e por maior segurança em relação aos efeitos das oscilações dos mercados (ELLIS, 2000a, 2000b).

No meio rural, a diversificação da produção pode ser observada de maneiras distintas, considerando tanto as atividades desenvolvidas dentro da propriedade rural quanto também as realizadas fora do estabelecimento agrícola (ILBERY, 1991; ELLIS, 2000a, 2000b; ABDULAI; CROLEREES, 2001; BARBIERI; MAHONEY, 2009; BARBIERI; MSHENGA, 2008; PLOEG; ROEP, 2003; VIK; MCELWEE, 2011; MERANER et al., 2015). Este estudo considera a diversificação do ponto de vista dos recursos agrícolas (terra, capital e trabalho) alocados para o desenvolvimento de atividades realizadas dentro da propriedade rural com foco na agricultura, que possam abranger o processamento e o aprimoramento de produtos, a agregação de valor e a venda de produtos na propriedade (ILBERY, 1991; TURNER et al., 2003; BARBIERI; MAHONEY, 2009; PLOEG; ROEP, 2003).

Estudos têm apontado que características como idade (BARBIERI; MAHONEY, 2009), disponibilidade de mão de obra familiar (HJALAGER, 1996; NILSSON, 2002; MISHRA; EL-OSTA; SANDRETTO, 2004; BENJAMIN; KIMHI, 2006), tamanho da propriedade rural (ILBERY, 1991; HJALAGER, 1996; MCNALLY, 2001; MISHRA; EL-OSTA; SANDRETTO, 2004; VIK; MCELWEE, 2011), tipo de atividade desenvolvida na propriedade (MCNALLY, 2001; JONGENEEL; POLMAN; SLANGEN, 2008) e características geográficas (ILBERY, 1991; MISHRA; EL-OSTA; SANDRETTO, 2004; BARBIERI; MAHONEY, 2009; PFEIFER et al., 2009; LANGE et al., 2013), são aspectos que influenciam a decisão de diversificar a produção agrícola nas propriedades rurais.

Além disso, outros estudos envolvendo o tema da diversificação abordam as influências comportamentais relacionadas aos motivos ou valores dos agricultores referentes à tal tomada de decisão. Vik e McElwee (2011), ao analisarem as motivações para a diversificação agrícola na Noruega, verificaram que os motivos sociais são tão importantes quanto os econômicos e que as motivações variam de acordo com os diferentes tipos de diversificação. Por sua vez, Hansson et al. (2013) observaram a influência da família sobre os motivos dos agricultores em diversificar suas atividades além das tarefas agrícolas, e constataram que existem razões associadas à redução de risco e utilização de recursos ociosos, além de motivações sociais e de estilo de vida. Ferguson e Hansson (2013) verificaram como os valores dos produtores rurais influenciam seus planos de expandir, manter ou parar sua produção de leite.

Neste contexto, pesquisas no meio rural com enfoque da psicologia social, principalmente utilizando a Teoria do Comportamento Planejado (TCP), têm sido cada vez mais frequentes (BURTON, 2004). Segundo a TCP, a intenção é o principal preditor de um comportamento e é determinada pelos constructos de atitude, norma subjetiva e controle comportamental. A utilização destes três constructos permite identificar como 
os agricultores avaliam a possibilidade de diversificar a produção agrícola em suas propriedades (constructo de atitude), verificar a função da pressão social percebida pelos agricultores para diversificar a produção (constructo de norma subjetiva) e identificar a percepção dos agricultores quanto à sua capacidade de utilizar esta estratégia produtiva em seus estabelecimentos rurais (constructo de controle comportamental percebido).

Por sua vez, em seu estudo, Dalcin (2013) observou que o desempenho econômico das propriedades rurais é influenciado pelas características do agricultor, da propriedade, pelo estilo de tomada de decisão e pelo comportamento empreendedor ou não empreendedor. Ao estudar o estilo de decisão dos produtores rurais, Dutra (2008) constatou a existência de dois grupos distintos: um de produtores com estilo predominantemente hierárquico e outro composto por produtores rurais com estilo integrativo. Desta forma, as lacunas existentes nas pesquisas anteriores possibilitam a realização de um estudo que integre os estilos de decisão e diferentes níveis de intenção em diversificar a produção.

Considerando que nos estudos sobre a diversificação da produção nas propriedades rurais predomina a abordagem econômica, este estudo se diferencia dos demais por utilizar a Teoria do Comportamento Planejado (TCP) como abordagem teórico-metodológica. Dentre os estudos que utilizaram a TCP para analisar a diversificação, este se distingue por utilizar os diferentes níveis de intenção em suas análises. Seu emprego se justifica primeiramente pelo crescimento deste tipo de enfoque, o qual sugere que o comportamento dos agricultores não é acionado apenas pela maximização do lucro (GASSON, 1973) e também porque poucos estudos têm sido realizados utilizando a psicologia social nas tomadas de decisão pela diversificação das atividades agrícolas nas pequenas propriedades (HANSSON; FERGUNSON; OLOFSSON, 2012; MARTÍNEZ-GARCÍA; DORWARD; REHMAN, 2013). Assim, o objetivo deste estudo consiste em analisar se os diferentes níveis de intenção dos agricultores familiares em diversificar a produção agrícola podem ser explicados pelos constructos Atitude, Norma Subjetiva e Controle Comportamental Percebido, pelos estilos de decisão e pelas características socioeconômicas dos agricultores.

\section{A TEORIA DO COMPORTAMENTO PLANEJADO (TCP) COMO ABORDAGEM TEÓRICO-METODOLÓGICA}

A Teoria do Comportamento Planejamento (TCP) foi proposta, em 1985, pelo psicólogo social Icek Ajzen como sendo uma derivação da Teoria da Ação Racional, proposta por Ajzen e Fishbein (1980). Desde então, esta abordagem vem sendo amplamente utilizada, tornando-se um referencial teórico-metodológico útil para compreender a intenção e a tomada de decisão na agricultura, abrangendo temas como conservação (BEEDELL; REHMAN, 2000), empreendedorismo (BERGEVOET et al., 2004), gestão de áreas ribeirinhas (FIELDING et al., 2005), práticas agrícolas sustentáveis (FIELDING et al., 2008), mortalidade de cordeiros (ELLIOTT et al., 2011), diversificação (HANSSON; FERGUNSON; OLOFSSON, 2012), práticas de bem-estar animal (LAUWERE et al., 2012), propriedades rurais orgânicas (LÄPPLE; KELLEY, 2013), uso de pastagem natural melhorada (BORGES et al., 2014) e práticas de conservação da água (YAZDANPANAH et al., 2014). 
A TCP pressupõe que quanto mais forte a intenção de se envolver em um comportamento, mais provável deve ser o seu desempenho (AJZEN, 1991, 2005). Para esta teoria, as intenções comportamentais são originadas de três constructos independentes conceitualmente: atitude, norma subjetiva e controle comportamental percebido, os quais podem ser mensurados diretamente ou ainda derivar das crenças dos indivíduos (medidas indiretas).

A atitude refere-se ao grau com que uma pessoa tem uma avaliação favorável ou desfavorável em relação a um comportamento (AJZEN, 1991). O segundo constructo, denominado norma subjetiva, é um fator social que corresponde à pressão social percebida para manifestar ou não o comportamento. O terceiro é o grau de controle comportamental observado, que equivale à facilidade ou dificuldade percebida pelo indivíduo em exibir o comportamento (AJZEN, 1991).

A utilização destes três constructos permite identificar como os agricultores avaliam a possibilidade de diversificar a produção agrícola em suas propriedades (constructo de atitude), verificar a função da pressão social percebida pelos agricultores para diversificar a produção (constructo de norma subjetiva) e identificar a percepção dos agricultores quanto à sua capacidade de utilizar esta estratégia produtiva em seus estabelecimentos rurais (constructo de controle comportamental percebido). Ajzen (1991) esclarece que, quanto mais favoráveis forem estes três constructos, mais forte deve ser a intenção de um indivíduo manifestar o comportamento analisado. Nesse contexto, o presente estudo possui duas hipóteses de pesquisa. A primeira delas é a $\mathrm{H} 1$, quando agricultores com intenção mais forte de diversificar a produção agrícola em suas propriedades têm valores mais altos para atitude, norma subjetiva e controle comportamental percebido, comparados com os agricultores que têm intenção mais fraca.

\section{ESTILOS DECISÓRIOS NA TOMADA DE DECISÃO}

A literatura apresenta que a tomada de decisão tem sido influenciada por elementos diferenciados, como juízo, princípios e valores das pessoas, negociação política, senso comum e características socioeconômicas e psicológicas, o que não significa a total exclusão da racionalidade gerencial, apenas uma ampliação de conceitos (GASSON, 1973; BURTON, 2004; PARKER; FISCHHOFF, 2005; MACHADO; OLIVEIRA; SCHNORRENBERGER, 2006).

Da mesma forma que existem diferentes tipos de decisão, há distintos estilos de tomadas de decisão, os quais interferem no percurso das atividades no dia a dia dos estabelecimentos rurais. Segundo Jamian, Sidhu e Aperapar (2013), o estilo de tomada de decisão é um fator-chave que contribui tanto para o sucesso dos gestores quanto das organizações. Na concepção de Rowe e Boulgarides (1983), Rowe e Mason (1987) e Rowe, Mason e Dilckel (1998), os indivíduos agem sob a influência preponderante do hemisfério esquerdo ou do hemisfério direito do cérebro, existindo, assim, duas dimensões que diferenciam os tomadores de decisão: uma orientada para a tarefa (com foco no desempenho) e outra para as pessoas (com foco nos relacionamentos interpessoais) (ROWE; MASON; DILCKEL, 1998). Segundo estes autores, da combinação dessas duas dimensões resultam quatro estilos de tomada de decisão: diretivo, comportamental, analítico e conceitual, os quais se baseiam essencialmente em duas dimensões do pensamento: complexidade cognitiva e orientação dos valores. 
Robbins (2006) salienta que, mesmo que cada um destes quatro estilos seja distinto, os gestores podem apresentar características pertencentes a mais de um deles; por isso, pode-se considerar que os indivíduos devem apresentar um estilo dominante. Além disso, em virtude das particularidades de cada setor da economia, pode ser que um determinado estilo de decisão se destaque perante os demais. Torna-se, assim, pertinente identificar e verificar como isso se comporta na agricultura.

Com a finalidade de identificar e medir estes quatro estilos de tomada de decisão dos indivíduos, Rowe e Mason (1987) desenvolveram o Decision Style Inventory (DSI), o qual foi utilizado neste estudo. Este instrumento tem sido utilizado para analisar os estilos de decisão associados a temas como gestão acadêmica (JAMIAN; SIDHU; APERAPAR, 2013), gestão pública (CONNOR; BECKER, 2003), uso de tecnologias (JACOBY, 2006), satisfação no trabalho (AMAZT; IDRIS, 2011), gestão de projetos (FOX; SPENCE, 1999), relação entre professor orientador e seus orientados (ARMSTRONG, 2004). Não foram identificados, entretanto, estudos com a aplicação do modelo DSI no meio rural. Assim sendo, surge a segunda hipótese deste estudo: $\mathrm{H} 2$, quando agricultores com diferentes níveis de intenção de diversificar a produção apresentam estilos de decisão distintos.

O estudo dos estilos decisórios torna-se útil na medida em que descreve o modo como os gestores tomam suas decisões ou a maneira pela qual eles reagem a uma determinada situação. Isso inclui o contexto sob o qual as decisões são tomadas, a forma como estes gestores percebem e interpretam as informações, o que eles valorizam e julgam essencial e como reagem às inúmeras forças do ambiente.

\section{MATERIAIS E MÉTODOS}

\section{Medindo os Constructos da TCP}

Inicialmente foi definido que o comportamento analisado neste estudo seria a intenção dos agricultores em diversificar a produção agrícola em suas propriedades rurais. Os constructos da TCP foram medidos utilizando-se escalas de cinco pontos, com o valor um sendo atribuído a respostas negativas e o valor cinco para respostas associadas positivamente. Escalas de cinco pontos têm sido usadas em estudos que abrangem o meio agrícola (BARBIERI; MAHONEY, 2009; FERGUSON; HANSSON, 2015; HANSSON et al., 2013), pois podem ser consideradas curtas o suficiente para os respondentes distinguirem entre as opções de resposta (HANSSON; FERGUSON; OLOFSSON., 2012).

A intenção dos agricultores em diversificar a produção foi medida pelo cálculo da média de quatro declarações. Como os constructos atitude, norma subjetiva e controle comportamental podem ser medidos de forma direta ou indireta, optou-se por utilizar ambas as medidas neste estudo. A atitude direta foi mensurada pela média de outras cinco declarações. Para a norma subjetiva direta utilizou-se a média das respostas de três declarações. O controle comportamental percebido, medido diretamente, foi obtido pelo cálculo da média de cinco declarações.

Para identificar as variáveis associadas às possíveis consequências, os prováveis referentes importantes e os fatores que facilitam ou dificultam a diversificação da produção, as quais foram utilizadas para medir indiretamente os constructos da TCP, realizaram-se entrevistas semiestruturadas com nove agricultores. Utilizou-se a análise de 
conteúdo para interpretação das entrevistas. Cabe salientar que tais entrevistas serviram única e exclusivamente para a identificação das variáveis, incluídas posteriormente no questionário. Estes agricultores foram indicados por extensionistas da Associação Riograndense de Empreendimentos de Assistência Técnica e Extensão Rural (Emater/ RS) do município de Frederico Westphalen. Julgou-se que o perfil destes agricultores representou os demais agricultores da região. Destaca-se que tal procedimento foi adotado e realizado seguindo os pressupostos de Ajzen (1991).

O modelo apresentado pela TCP pressupõe que a medida da atitude $(A)$, em razão das possíveis consequências $(i)$, considera a intensidade da consequência $\left(c_{i}\right)$ e a avaliação das consequências $\left(a_{i}\right)$. Desta forma, para cada possível consequência $i$, elaboraram-se duas perguntas, as quais foram respondidas com base em uma escala de cinco pontos. Primeiramente, para medir $c_{i}$, cada consequência foi apresentada na forma de vantagens ou desvantagens, normalmente relacionadas com a diversificação da produção nas propriedades rurais, buscando-se identificar o grau de concordância dos entrevistados na escala fornecida (1: Discordo totalmente - 5: Concordo totalmente). Posteriormente, para a identificação $a_{i}$, foi utilizada uma escala para medir o grau de importância (1: Extremamente sem importância - 5: Extremamente importante). Assim, para cada $i$ foi calculado o produto entre $c_{i}$ e $a_{i}$, resultando em sete crenças comportamentais $\left(\mathrm{c}_{\mathrm{i}} \cdot a_{i}\right)$. A atitude indireta resultou da soma destas crenças $\left(A \propto \sum_{n} c_{i} . a_{i}\right)$.

A norma subjetiva (NS) resulta dos referentes importantes (j). Sua mensuração é consequência da multiplicação entre a intensidade do referente importante $(j)\left(c_{j}\right)$ e a motivação de cada respondente em considerá-lo $\left(m_{j}\right)$. Sendo assim, para cada referente importante $j$ foram elaboradas duas perguntas, nas quais os respondentes utilizaram uma escala de cinco pontos para responder. Primeiramente, para medir $\mathrm{c}_{\mathrm{j}}$, foi apresentada a seguinte questão: “Caso você decida diversificar a produção agrícola pelo menos em alguma das atividades desenvolvidas na sua propriedade nos próximos cinco anos, você acha que [referente importante $(j)$ ] (1: Reprovaria totalmente - 5: Aprovaria totalmente)?". Em seguida, para medir $m_{j}$ fez-se a seguinte colocação: "Quanto você se importa com o que pensa [referente importante $(j)$ ] sobre o que você deveria fazer em sua propriedade (1: Não me importo nada - 5: Me importo muito)?". De maneira semeIhante às atitudes, para cada referente importante $j$ foi calculado o produto entre $c_{j}$ e $m_{j^{\prime}}$ resultando em oito crenças normativas, as quais foram somadas $\left(N S \propto \sum_{n} c_{j} . m_{j}\right.$ ) para obter a norma subjetiva indireta de cada indivíduo entrevistado.

Por sua vez, identificou-se ainda o controle comportamental percebido, que resulta dos possíveis fatores $(k)$. Para sua mensuração, foi efetuada a multiplicação entre a intensidade de cada fator $\left(c_{k}\right)$ e a potência percebida de cada fator $\left(p_{k}\right)$ para facilitar ou inibir o desempenho do comportamento. Para cada um dos fatores $(k)$, foram elaboradas duas perguntas, ambas medidas com escalas de cinco pontos. A primeira para medir $c_{k}:$ "Qual seu nível de concordância para [possível fator $(k)$ ], que pode facilitar ou dificultar que você diversifique a produção agrícola pelo menos em alguma das atividades desenvolvidas na sua propriedade rural nos próximos cinco anos (1: Discordo totalmente - 5: Concordo totalmente)?". E a segunda para medir $p_{k}$ : "Qual a importância de [possível fator $(k)$ ] para que você diversifique a produção agrícola pelo menos em alguma das atividades desenvolvidas na sua propriedade rural nos próximos cinco anos (1: Extremamente sem importância - 5: Extremamente importante)?". Os resulta- 
dos desses produtos $\left(c_{k}, p_{k}\right)$ resultaram em 13 crenças de controle comportamental, as quais foram somadas $\left(C C P \propto \sum_{n} c_{k} \cdot p_{k}\right)$ para se obter o controle comportamental percebido indireto de cada produtor rural.

\section{Identificando o Estilo de Decisão e as Características Socioeconômicas}

O DSI foi utilizado para identificar a propensão de um indivíduo para cada estilo de decisão (diretivo, analítico, conceitual e comportamental) dos agricultores. O nível de intensidade de cada um dos quatro estilos de decisão permite classificar os estilos decisórios em Menos Preferido, Back-Up, Dominante e Muito Dominante.

A intensidade Menos Preferido sugere que o indivíduo raramente utiliza aquele estilo, mas, quando for necessário, pode fazê-lo. A intensidade Back-Up indica que o indivíduo utilizará o respectivo estilo eventualmente. Por sua vez, o indivíduo que apresenta intensidade Dominante demonstra que frequentemente usa o referido estilo de decisão. Para Rowe e Mason (1987), os indivíduos podem apresentar mais de um estilo dominante, sendo possível alternar entre um e outro. O quarto nível de intensidade se refere ao Muito Dominante, indicando o estilo preferido pelos indivíduos, o qual é usado compulsivamente no processo decisório.

Para tanto, o modelo compreende 20 frases iniciais, relacionadas a situações enfrentadas pelos gestores, e quatro possíveis opções para finalizá-las. Em cada uma destas questões os entrevistados devem classificar cada uma das quatro opções de resposta, seguindo a seguinte escala: oito - indica que a resposta é a mais parecida com ele; quatro - indica que a resposta é moderadamente parecida com ele; dois - indica que a resposta é um pouco parecida com ele; e um - indica que a opção é a menos parecida com ele.

O escore final de cada estilo de decisão foi obtido somando-se o resultado das 20 respostas respectivas de cada estilo de decisão atribuídas pelos respondentes. Este escore permitiu a classificação dos agricultores seguindo os critérios apresentados na Tabela 1. Também se utilizou o escore final de cada estilo de decisão para comparar os grupos de diferente intenção de diversificação.

Tabela 1 - Níveis de intensidade dos estilos de decisão

\begin{tabular}{lcccc}
\hline Estilo de decisão & Menos Preferido & Back-Up & Dominante & Muito Dominante \\
\hline Diretivo & Abaixo de 68 & 68 a 82 & 83 a 90 & Acima de 90 \\
Analítico & Abaixo de 83 & 83 a 97 & 98 a 104 & Acima de 104 \\
Conceitual & Abaixo de 73 & 73 a 87 & 88 a 94 & Acima de 94 \\
Comportamental & Abaixo de 48 & 48 a 62 & 63 a 70 & Acima de 70 \\
\hline
\end{tabular}

Fonte: ROWE; BOULGARIDES (1992).

\section{Amostragem e Procedimentos para Coleta de Dados}

A presente pesquisa caracteriza-se como sendo de cunho predominantemente quantitativo, uma vez que emprega a pesquisa qualitativa apenas para a identificação de variáveis a serem medidas posteriormente por um questionário. Este estudo foi realizado na Região Noroeste do Estado do Rio Grande do Sul, Brasil, especificamente no município de Frederico Westphalen. Decidiu-se, primeiramente, por identificar agricul- 
tores especializados na produção de leite. Optou-se por esta atividade pelo fato de ela ser uma das mais presentes no meio rural da região pesquisada, contribuindo, assim, para o sustento das famílias dos agricultores.

Para fins da pesquisa, foi utilizada a participação das atividades rurais na renda bruta da propriedade como critério para diferenciar os estabelecimentos rurais especializados dos diversificados (HANSSON; FERGUNSON; OLOFSSON, 2010). Desta forma, se $50 \%$ ou mais da renda forem originários de uma única atividade rural, o estabelecimento agrícola foi considerado especializado, e, quanto maior for este valor, pode-se afirmar que maior é sua especialização (HOFFMANN et al., 1987). De posse de um relatório fornecido pela Prefeitura de Frederico Westphalen, verificou-se a existência de 460 produtores rurais que, de alguma forma, comercializaram leite durante o ano de 2013. Com a ajuda dos técnicos extensionistas agrícolas da Emater/RS, foram identificados 120 agricultores especializados na produção de leite para compor a população deste estudo.

Caso o agricultor apresentasse algum sinal de resistência para responder, ou não atendesse o perfil desejado, não se realizaria a pesquisa com ele. Em alguns casos, o agricultor indicava outro produtor agrícola, muitas vezes alguém na sua própria localidade, que atendesse as necessidades da pesquisa. Desta forma, foram visitados por um entrevistador 101 estabelecimentos rurais, que representam 22\% das propriedades agrícolas produtoras de leite, ou $84 \%$ das propriedades que possuem $50 \%$ ou mais da renda oriunda desta atividade rural. As informações foram coletadas nos meses de novembro e dezembro de 2014.

\section{ANÁLISE DOS DADOS}

A intenção comportamental foi obtida pela média das quatro questões da intenção. O coeficiente Alfa de Cronbach para o conjunto de perguntas deste constructo correspondeu a 0,894, acima do mínimo recomendado (HAIR et al., 2010; MALHOTRA, 2009), indicando que os resultados das variáveis utilizadas para identificar a intenção podem ser somados e que a média pode ser usada para representar este constructo (BORGES et al., 2014).

A divisão mediana ( $\mathrm{Md}=2,5)$ foi realizada na escala dos valores da média das quatro questões da intenção (FIELDING et al., 2005), com a finalidade de formar um grupo com intenção fraca $(n=64, \bar{x}=1,7)$ e outro com intenção forte $(n=37, \bar{x}=3,9)$ de diversificar a produção agrícola.

Para as variáveis categóricas, usadas para caracterizar o perfil dos entrevistados, utilizou-se a distribuição de frequência, medidas de tendência central (média aritmética, moda, mediana) e medidas de variabilidade (limites mínimos, máximos, coeficiente de variação, variância e desvio-padrão).

Depois de identificados estes dois grupos, buscou-se verificar se os constructos psicológicos propostos pela TCP seriam capazes de explicar os diferentes níveis de intenção dos agricultores. As diferenças entre os grupos foram testadas usando o teste de Mann-Whitney para as variáveis ordinais. 


\section{RESULTADOS E DISCUSSÃO}

\section{Os Constructos da TCP e suas Medidas Diretas e Indiretas}

Os resultados do teste de Mann-Whitney (Tabela 2) reforçam a existência de dois grupos diferentes entre si nos constructos da TCP medidos diretamente. Dos 101 produtores rurais entrevistados, identificou-se um grupo com fraca intenção de diversificar a produção, composto por 53 produtores (52,5\%), e outro grupo com forte intenção de diversificar, sendo formado por 48 agricultores (47,5\%). Verificou-se que o grupo dos agricultores com fraca intenção pela diversificação apresentou valores menores de atitude direta, norma subjetiva direta e controle comportamental percebido do que o grupo dos agricultores com forte intenção. Além disso, os produtores rurais com fraca intenção de diversificar suas atividades agrícolas tiveram valores significativamente mais baixos para atitude indireta e norma subjetiva indireta. Os resultados, entretanto, evidenciaram que os grupos não diferem significativamente entre si quando analisado o constructo de controle comportamental percebido medido indiretamente.

Desta forma, rejeitou-se parcialmente a hipótese $\mathrm{H}_{1}$ : Agricultores com intenção mais forte de diversificar a produção agrícola em suas propriedades têm valores mais altos para atitude, norma subjetiva e controle comportamental percebido, comparados com os agricultores que têm intenção mais fraca. Tal hipótese foi rejeitada para as medidas indiretas do controle comportamental percebido.

Comparando os dois grupos por meio dos constructos da TCP medidos diretamente, constatou-se que os agricultores com forte intenção avaliaram mais favoravelmente a diversificação nas suas propriedades do que os com fraca intenção (atitude direta).

Tabela 2 - Média das medidas diretas e indiretas dos constructos da TCP para cada um dos dois grupos de intenção

\begin{tabular}{lcc}
\hline & \multicolumn{2}{c}{ Intenção de diversificar } \\
& Fraca & Forte \\
& $(\mathrm{n}=53)$ & $(\mathrm{n}=48)$ \\
\hline Intenção $^{\mathrm{a}}$ & 1,7 & 3,9 \\
Atitude Direta $^{\mathrm{a}}$ & 3,1 & 4,1 \\
Norma Subjetiva Direta $^{\mathrm{a}}$ & 2,8 & 3,7 \\
Controle Comportamental Percebido Direto $^{\mathrm{a}}$ & 2,6 & 3 \\
Atitude Indireta $^{\mathrm{a}}$ & 8 & 11,1 \\
Norma Subjetiva Indireta $^{\mathrm{a}}$ & 11,6 & 13,4 \\
Controle Comportamental Percebido Indireto & 4,7 & 4,6 \\
\hline
\end{tabular}

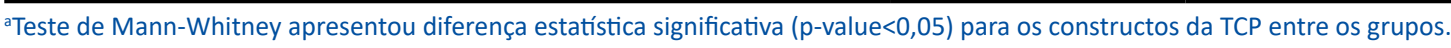

Fonte: Dados da Pesquisa.

Da mesma forma, o grupo de agricultores com forte intenção percebe que sofre maior pressão social para diversificar sua produção agrícola quando comparado com o grupo de intenção fraca (norma subjetiva direta). Os agricultores com fraca intenção de diversificar a produção agrícola demonstraram uma capacidade de diversificar a produção na sua propriedade mais fraca que o grupo de forte intenção (controle comportamental percebido direto). 
Os resultados deste estudo são parcialmente consistentes com os de Borges e Lansink (2015), os quais encontraram que agricultores com níveis de intenção mais altos para utilizar pastagem natural melhorada apresentaram valores mais altos para os três constructos da TCP, medidos tanto de forma direta quanto indireta.

Agricultores com forte e fraca intenção mostraram ainda diferenças em suas crenças comportamentais sobre os resultados relacionados com a diversificação, suas crenças normativas relacionadas aos outros referentes importantes e suas crenças de controle que abrangem os fatores que podem facilitar ou inibir a diversificação nas suas propriedades. Os resultados desta pesquisa corroboram os de Borges e Lansink (2015).

\section{Crenças Comportamentais}

Os resultados apresentados na Tabela 3 mostram que agricultores com forte e fraca intenção diferem em suas crenças comportamentais. Os dois grupos apresentaram percepções distintas sobre a probabilidade do resultado $\left(c_{i}\right)$ e avaliação do resultado $\left(a_{i}\right)$. Os agricultores com forte intenção classificam cinco crenças comportamentais como sendo os resultados mais prováveis da diversificação da produção, as quais diferem significativamente dos resultados apresentados pelo grupo com fraca intenção.

O grupo de agricultores com forte intenção de diversificar relatou que é menos provável que irá perder o controle das atividades já desenvolvidas na sua propriedade caso diversifique sua produção agrícola. Este grupo também evidenciou que é menos provável a necessidade de contratar empregados para desenvolver outras atividades ou aumentar a produção das já existentes e que terá de trabalhar muito mais para isso. Por outro lado, o grupo com forte intenção de diversificar, quando comparado com o grupo de fraca intenção, considerou mais provável que esta estratégia produtiva contribuirá para "manter os jovens na propriedade" e "ter mensalmente uma fonte renda".

Tabela 3 - Média das crenças comportamentais para cada um dos dois grupos de intenção

\begin{tabular}{|c|c|c|c|c|}
\hline \multirow{2}{*}{ Consequências } & \multicolumn{2}{|c|}{ Probabilidade do resultado $\left(c_{i}\right)$} & \multicolumn{2}{|c|}{ Avaliação do resultado $\left(a_{i}\right)$} \\
\hline & $\begin{array}{c}\text { Intenção } \\
\text { fraca }\end{array}$ & $\begin{array}{l}\text { Intenção } \\
\text { forte }\end{array}$ & $\begin{array}{l}\text { Intenção } \\
\text { fraca }\end{array}$ & $\begin{array}{l}\text { Intenção } \\
\text { forte }\end{array}$ \\
\hline $\begin{array}{l}\text { Perder o controle das atividades já } \\
\text { desenvolvidas }{ }^{\text {ab }}\end{array}$ & 2,6 & 3,8 & 1,7 & 1,7 \\
\hline Comprar máquinas/equipamentos ${ }^{a}$ & 2,3 & 2,5 & 2,2 & 1,9 \\
\hline Contratar empregados ${ }^{\mathrm{ab}}$ & 2,5 & 3,3 & 3,2 & 3,3 \\
\hline Desnecessário comprar mais terras & 2,4 & 2,8 & 3,2 & 3,8 \\
\hline Manter os jovens na propriedade ${ }^{b}$ & 2,5 & 3,6 & 4,4 & 4,7 \\
\hline $\begin{array}{l}\text { Ter mensalmente uma fonte de } \\
\text { rendab }\end{array}$ & 3,4 & 4,8 & 4,9 & 4,8 \\
\hline Ter de trabalhar muito mais ${ }^{\mathrm{ac}}$ & 1,3 & 2,2 & 2,2 & 2,7 \\
\hline
\end{tabular}

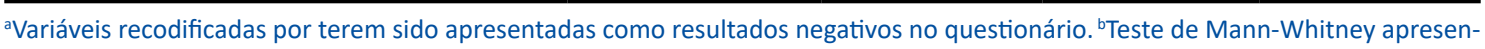
tou diferença estatística significativa $(\mathrm{p}$-value $<0,05)$ para $c_{i}{ }^{\mathrm{c}}$ Teste de Mann-Whitney apresentou diferença estatística significativa (p-value<0,05) para $c_{i}$ e $a_{i}$. 
A probabilidade percebida dos resultados não apresentou diferença estatisticamente significativa entre os grupos para duas crenças comportamentais ("comprar máquinas/equipamentos" e "desnecessário comprar mais terras"). Isto demonstra que estas duas crenças são incapazes de distinguir os grupos de agricultores de fraca e forte intenção.

Considerando a avaliação dos resultados provenientes da diversificação da produção agrícola, a única que apresentou diferença significativa entre os grupos foi "ter que trabalhar muito mais", evidenciando que os agricultores com forte intenção de aumentar a produção das atividades agrícolas complementares à produção de leite ou de investir em outras atividades, atribuíram maior importância ao fato de terem de trabalhar muito mais se comparados com os agricultores com fraca intenção de diversificar.

Os resultados desta pesquisa demonstraram que tanto algumas consequências positivas quanto negativas, provenientes da diversificação, foram capazes de diferenciar os grupos. Isto contrasta parcialmente com os achados de Fielding et al. (2005), que, em um estudo com agricultores da Austrália, verificaram que os grupos de produtores com fraca ou forte intenção diferem em suas crenças comportamentais a respeito dos resultados positivos, mas não em suas crenças sobre os resultados negativos. Considerando especificamente as crenças comportamentais, os resultados deste estudo trazem algumas implicações para os formuladores de políticas públicas, agentes de extensão e outras organizações envolvidas com os agricultores. A elaboração de ações junto aos agricultores da região estudada, principalmente com os de fraca intenção, que reforcem os resultados positivos da diversificação da produção, parece ser uma estratégia válida para aumentar a intenção dos produtores em diversificar.

\section{Crenças Normativas}

As diferenças estatísticas existentes entre os grupos de fraca e forte intenção para as crenças normativas são apresentadas na Tabela 4. Verificou-se que estes grupos diferem quanto às expectativas normativas dos outros considerados importantes. $O$ grupo com forte intenção de diversificar pensa ser mais provável do que o grupo de fraca intenção que "seus familiares", "agricultores vizinhos" e "amigos" irão aprovar totalmente caso eles decidam pela diversificação agrícola.

A motivação em considerar a opinião de outros referentes importantes sobre o que cada agricultor deve ou não fazer em sua propriedade não apresentou diferença entre os grupos com distintas intenções de diversificação. Cabe considerar que ambos os grupos se importam com a opinião destes agentes, principalmente com o que pensam seus familiares, os técnicos da empresa compradora de leite, a Emater/RS e a Cooperativa Tritícola de Frederico Westphalen/RS (Cotrifred).

Assim, ficou evidente a influência da família, dos agricultores vizinhos e dos amigos na decisão dos agricultores em diversificar, tanto que as expectativas normativas dos outros importantes diferenciaram os grupos de fraca e forte intenção. Gasson e Potter (1988) também ressaltam o papel significativo que as discussões familiares têm na compreensão do processo de decisão nas propriedades rurais. Os resultados desta pesquisa demonstram que a intenção de diversificar as atividades agrícolas aumentará se as ações sugeridas anteriormente forem feitas envolvendo não somente o agricultor, mas, principalmente, seus familiares. 
Tabela 4 - Média das crenças normativas para cada um dos dois grupos de intenção

\begin{tabular}{|c|c|c|c|c|}
\hline \multirow{2}{*}{ Referentes importantes } & \multicolumn{2}{|c|}{$\begin{array}{l}\text { Expectativas normativas de } \\
\text { outros importantes }\left(c_{j}\right)\end{array}$} & \multicolumn{2}{|c|}{$\begin{array}{l}\text { Motivação em } \\
\text { considerá-la }\left(m_{j}\right)\end{array}$} \\
\hline & $\begin{array}{c}\text { Intenção } \\
\text { fraca }\end{array}$ & $\begin{array}{l}\text { Intenção } \\
\text { forte }\end{array}$ & $\begin{array}{l}\text { Intenção } \\
\text { fraca }\end{array}$ & $\begin{array}{l}\text { Intenção } \\
\text { forte }\end{array}$ \\
\hline Seus familiares ${ }^{a}$ & 3 & 4 & 3,7 & 4,1 \\
\hline Prefeitura (Sec. Agricultura) & 3,7 & 3,9 & 2,9 & 3,2 \\
\hline Técnicos da empresa compradora de leite & 3 & 3,1 & 3,8 & 3,8 \\
\hline Emater/RS & 3,7 & 3,9 & 3,6 & 3,6 \\
\hline Agricultores vizinhos ${ }^{a}$ & 3,2 & 3,7 & 3 & 3 \\
\hline $\begin{array}{l}\text { Técnicos das empresas compradoras de outros } \\
\text { produtos (fumo, suínos, grãos, etc.) }\end{array}$ & 3,6 & 3,8 & 3 & 3,3 \\
\hline Cotrifred (Cooperativa) & 3,9 & 4 & 3,6 & 3,6 \\
\hline Amigos $^{a}$ & 3,2 & 3,8 & 3 & 3,4 \\
\hline
\end{tabular}

${ }^{a}$ Teste de Mann-Whitney apresentou diferença estatística significativa ( $p$-value $\left.<0,05\right)$ para $c_{j}$.

Fonte: Dados da Pesquisa.

\section{Crenças de Controle}

Os resultados apresentados na Tabela 5 comparam as médias das crenças de controle entre os grupos. Pode-se verificar que o grupo dos agricultores com fraca intenção difere do grupo de forte intenção quanto à probabilidade de os fatores estarem presentes para facilitar ou dificultar a diversificação agrícola apenas no fator "necessidade de mais conhecimento/atualizar-se para poder diversificar". Comparado com os agricultores de fraca intenção, o grupo dos agricultores com forte intenção percebe que é mais provável que a necessidade de adquirir mais conhecimento é um fator que está presente na agricultura como inibidor da diversificação da produção.

Comparando os grupos pela potência apresentada por crença para inibir ou facilitar a estratégia de diversificação, verificou-se que os agricultores com fraca intenção diferem dos de forte intenção apenas na crença "agricultores organizados para trabaIhar em conjunto na sua localidade". Agricultores com forte intenção de diversificar as atividades desenvolvidas na sua propriedade percebem que o poder deste fator para facilitar a diversificação é maior.

Tabela 5 - Média das crenças de controle para cada um dos dois grupos de intenção

\begin{tabular}{|c|c|c|c|c|}
\hline \multirow{2}{*}{ Fatores } & \multicolumn{2}{|c|}{$\begin{array}{l}\text { Probabilidade de o fator } \\
\text { estar presente }\left(c_{k}\right)\end{array}$} & \multicolumn{2}{|c|}{$\begin{array}{l}\text { Potência percebida de } \\
\text { cada crença }\left(p_{k}\right)\end{array}$} \\
\hline & $\begin{array}{c}\text { Intenção } \\
\text { fraca }\end{array}$ & $\begin{array}{l}\text { Intenção } \\
\text { forte }\end{array}$ & $\begin{array}{l}\text { Intenção } \\
\text { fraca }\end{array}$ & $\begin{array}{l}\text { Intenção } \\
\text { forte }\end{array}$ \\
\hline $\begin{array}{l}\text { Agricultores organizados para trabalhar em conjunto } \\
\text { na sua localidade }\end{array}$ & 2,3 & 2 & 3,7 & 4,2 \\
\hline $\begin{array}{l}\text { Realização de um projeto para orientar/acompanhar a } \\
\text { produção na propriedade }\end{array}$ & 2 & 2 & 1,7 & 1,4 \\
\hline Opções para venda e comercialização de produtos ${ }^{a}$ & 3,2 & 3,2 & 1,4 & 1,4 \\
\hline Motivação e idade avançada para trabalhar ${ }^{a}$ & 3,4 & 3,8 & 2,6 & 2,4 \\
\hline $\begin{array}{l}\text { Garantias para os agricultores investirem mais em } \\
\text { outras atividades }^{a}\end{array}$ & 1,8 & 1,7 & 1,4 & 1,3 \\
\hline
\end{tabular}




\begin{tabular}{|c|c|c|c|c|}
\hline $\begin{array}{l}\text { Ter mais gente da sua família para trabalhar na } \\
\text { propriedade }^{a}\end{array}$ & 2,3 & 2,3 & 2 & 1,8 \\
\hline Mais conhecimento/atualizar-se $\mathrm{e}^{\mathrm{ab}}$ & 2 & 1,6 & 1,5 & 1,2 \\
\hline Fazer financiamentos ${ }^{a}$ & 1,9 & 2 & 2,3 & 2,1 \\
\hline Terras que favorecem a produção agrícola ${ }^{a}$ & 3,4 & 3,7 & 1,3 & 1,4 \\
\hline Política de preços dos produtos ${ }^{\mathrm{a}}$ & 1,2 & 1,2 & 1,2 & 1,2 \\
\hline Legislação e a burocracia das atividades na agricultura & 1,9 & 1,7 & 3,4 & 3,7 \\
\hline Alto custo da produção agrícolaa & 1,4 & 1,5 & 2 & 1,7 \\
\hline Tempo para o investimento dar retorno ${ }^{a}$ & 2,1 & 2,4 & 1,6 & 1,6 \\
\hline
\end{tabular}

Fonte: Dados da Pesquisa.

Para analisar os seis fatores que dificultam a diversificação da produção agrícola, "necessidade de uma política de preços dos produtos agrícolas", "realização de um projeto para orientar/acompanhar a produção na propriedade", "alto custo da produção", "falta de garantias para os agricultores investirem mais em outras atividades", "fazer financiamentos" e "a realização de um projeto para orientar e acompanhar a produção na propriedade", a interpretação é um pouco diferente pelo fato de estas variáveis terem sido recodificadas.

Os dois grupos concordam em suas percepções de que a probabilidade de estes sete fatores estarem presentes no meio rural para inibir a diversificação é maior, e o poder percebido destes sete fatores em inibir a diversificação também foi maior. Os grupos concordam, ainda, que a probabilidade de "legislação e a burocracia para o desenvolvimento das atividades agrícolas" estarem presentes como um fator facilitador, é menor e atribuem a este fator maior poder para facilitar a diversificação das atividades rurais.

Os resultados das crenças de controle sugerem que se pode aumentar a intenção dos agricultores, especialmente a do grupo de fraca intenção, por meio de cursos de qualificação e aperfeiçoamento, bem como pela mobilização e organização dos agricultores para trabalharem em conjunto, uma vez que estes foram elementos destacados como facilitadores da diversificação.

\section{Estilo Decisório dos Agricultores Familiares}

A Tabela 6 demonstra a intensidade dos estilos de tomadas de decisão dos agricultores familiares que possuem na produção de leite a principal atividade geradora de renda na propriedade agrícola. Dentre os quatro estilos decisórios analisados, os resultados indicam que $90,1 \%$ dos agricultores pesquisados se percebem como tomadores de decisão essencialmente comportamentais, uma vez que $78,2 \%$ dos agricultores apresentaram intensidade muito dominante e outros $11,9 \%$ intensidade dominante para 0 estilo de decisão comportamental. 
Tabela 6 - Percentual de cada um dos níveis de estilo decisório $(n=101)$

\begin{tabular}{lcccc}
\hline Estilo de decisão & Menos Preferido & Back-Up & Dominante & Muito Dominante \\
\hline Diretivo & 40,6 & 35,6 & 14,9 & 8,9 \\
Analítico & 84,2 & 14,9 & 1 & 0 \\
Conceitual & 56,4 & 33,7 & 5,9 & 4 \\
Comportamental & prefer & 8,9 & 11,9 & 78,2 \\
\hline
\end{tabular}

Fonte: Dados da Pesquisa.

Os dados permitem concluir que prevaleceu o estilo comportamental para a maioria dos agricultores e que, em menor proporção, também existe uma parcela de produtores rurais que possuem estilo diretivo. Além disso, como previsto pela teoria, pode-se considerar ainda que um mesmo agricultor apresente igual nível de intensidade em mais de um estilo decisório, o que pode ser verificado principalmente nos percentuais do nível menos preferido, que excedeu $100 \%$.

Constatou-se também que $84,2 \%$ dos produtores rurais demonstraram que o estilo de decisão analítico é de intensidade menos preferida. Assim, constata-se que os agricultores têm pouca preferência por ambiguidade, buscar informações, lidar com situações novas, orientação para as tarefas e tomar decisões técnicas e racionais.

Para outros $56,4 \%$ o estilo de decisão conceitual foi considerado o menos preferido. Adicionalmente, os estilos de decisão diretivo e conceitual foram indicados por $35,6 \%$ e $33,7 \%$ dos entrevistados, respectivamente, como tendo intensidade de backup, o que significa que estes dois estilos de decisão poderão ser colocados em prática ocasionalmente.

\section{Análise Comparativa entre os Grupos de Diferentes Intenções com Base nas Características Socioeconômica e Estilos de Decisão}

Considerando as variáveis socioeconômicas apresentadas na Tabela 7, observou-se que os grupos de agricultores com fraca e forte intenção de diversificar a produção agrícola diferem pouco entre si. Dentre as variáveis socioeconômicas observadas, a quantidade de pessoas que compõe o grupo familiar e a área total de terras (em hectares) da propriedade rural foram capazes de diferenciar o grupo de agricultores de forte intenção do de fraca intenção de diversificar a produção. Constatou-se, ainda, que os agricultores com forte intenção de diversificar são aqueles que possuem grupo familiar maior, o que pode ser compreensível por apresentar maior disponibilidade de mão de obra.

Mishra, El-Osta e Sandretto (2004) e McNally (2001) afirmam que propriedades rurais com famílias maiores apresentam maior necessidade de diversificar quando comparadas com famílias menores, criando, assim, oportunidades de trabalho dentro da propriedade. Além disso, Meraner et al. (2015) verificaram que a força de trabalho familiar disponível apresenta um impacto significativamente positivo na probabilidade de diversificação. Benjamin e Kimhi (2006) corroboram estes resultados ao confirmarem que a ocorrência da diversificação das atividades agrícolas é mais provável nas propriedades rurais com disponibilidade de mão de obra feminina e crianças maiores. $\mathrm{O}$ tamanho da propriedade rural também é destacado pela literatura como um fator relacionado com a decisão de diversificar. 
Os resultados deste estudo demonstraram que o grupo com forte intenção de diversificar apresentou área total menor e significativamente diferente que a área do grupo com fraca intenção. Isto reforça os achados de Vik e McElwee (2011), que analisaram as motivações em prol da diversificação agrícola na Noruega, e também de Mishra, El-Osta e Sandretto (2004), que estudaram os fatores que afetam a diversificação agrícola nos Estados Unidos. Estes dois estudos constataram que propriedades rurais menores são mais prováveis de diversificar a produção agrícola. Por outro lado, o presente estudo contrasta com os resultados de Meraner et al. (2015), que estudaram a diversificação rural na Holanda, e com os achados de Ilbery (1991), que realizou seu estudo no Reino Unido. Ambos os estudos sugerem que propriedades rurais maiores afetam positivamente a probabilidade de diversificação.

Tabela 7 - Médias das variáveis socioeconômicas, estilos de decisão e orientação dos objetivos para os grupos com fraca e forte intenção de diversificar

\begin{tabular}{|c|c|c|}
\hline Variáveis & $\begin{array}{l}\text { Fraca intenção } \\
(n=53)\end{array}$ & $\begin{array}{c}\text { Forte intenção } \\
(n=48)\end{array}$ \\
\hline \multicolumn{3}{|l|}{ Características socioeconômicas } \\
\hline Quantidade de pessoas que compõem o grupo familiar & 3,3 & 3,7 \\
\hline Quantidade de filhos atuando na propriedade & 0,9 & 1,3 \\
\hline Idade & 50,4 & 46,9 \\
\hline Escolaridade & 2 & 2 \\
\hline Renda bruta mensal estimada totalc & 9.257 & 8.998 \\
\hline Renda bruta mensal estimada do leite ${ }^{c}$ & 6.825 & 6.461 \\
\hline Renda bruta mensal estimada das outras atividades ${ }^{c}$ & 2.453 & 2.394 \\
\hline Área total de terras para uso próprio ${ }^{a}$ & 23 & 17,6 \\
\hline Área destinada à atividade leiteira & 12 & 10 \\
\hline Participação percentual do leite na renda & 76,4 & 73,5 \\
\hline Tempo desenvolvendo atividades agrícolas & 37,7 & 35,1 \\
\hline Quantidade de atividades desenvolvidas & 1,8 & 2 \\
\hline \multicolumn{3}{|l|}{ Estilos decisórios } \\
\hline Diretivo & 72 & 71,3 \\
\hline Analítico & 69,8 & 70,1 \\
\hline Conceitual & 69 & 71,4 \\
\hline Comportamental ${ }^{\mathrm{b}}$ & 84 & 81,8 \\
\hline
\end{tabular}

a Teste de Mann-Whitney apresentou diferença estatística significativa ( $p$-value $<0,05$ ) entre os grupos. ${ }^{b}$ Estilo comportamental dos agricultores predominante nos dois grupos. ${ }^{\complement}$ Calculada com base nos dados de produção mensal de leite, participação do leite e das outras atividades na renda bruta da propriedade. Foi utilizada também a média dos valores nominais do preço do leite pago ao produtor no Estado do Rio Grande do Sul no ano de 2014, segundo o Cepea (2015).

Fonte: Dados da Pesquisa.

A idade dos agricultores familiares também foi observada. Apesar de o grupo dos agricultores com forte intenção de diversificar a produção agrícola ter apresentado idade menor quando comparado com o grupo de agricultores com fraca intenção, esta diferença foi estatisticamente insuficiente para distinguir um grupo do outro. Barbieri e Mahoney (2009) demonstraram que a idade do agricultor está associada de forma positiva com a diversificação, evidenciando que agricultores com mais idade tendem a 
diversificar. Os resultados de Mishra, El-Osta e Sandretto (2004) e Meraner et al. (2015) demonstraram que a idade tem um efeito negativamente significativo na decisão de diversificação, ou seja, que é mais provável que gestores mais jovens diversifiquem a produção agrícola em suas propriedades rurais; entretanto, tal resultado não pode ser corroborado nesta pesquisa.

Analisando os estilos de decisão, verificou-se que os grupos são semelhantes entre si, uma vez que nenhum dos quatro estilos de decisão pesquisados foi suficiente para distinguir o grupo de agricultores de fraca intenção de diversificar do de forte intenção. Constatou-se que o estilo de decisão comportamental foi o que prevaleceu nos dois grupos de agricultores. Sendo assim, rejeita-se a hipótese $\mathrm{H}_{2}$ : Agricultores com diferentes níveis de intenção de diversificar a produção apresentam estilos de decisão distintos.

\section{CONSIDERAÇÕES FINAIS}

Este estudo buscou explicar diferentes níveis de intenção dos agricultores em diversificar a produção agrícola em suas propriedades por meio dos fatores da psicologia social. Também foram analisadas as diferenças dos aspectos socioeconômicos e dos estilos de decisão dos grupos de produtores rurais com diferentes níveis de intenção.

Dois grupos de agricultores com diferentes níveis de intenção foram encontrados: um com fraca intenção de diversificar e outro com forte intenção. Agricultores com fraca e forte intenção distinguem-se entre si em termos de suas medidas diretas e indiretas de atitude e norma subjetiva. As medidas diretas de controle comportamental percebidas também apresentaram diferença entre os grupos, entretanto a medida indireta do controle comportamental foi incapaz de distinguir um grupo do outro. Agricultores familiares com diferentes níveis de intenção em diversificar a produção agrícola não apresentaram diferenças nos estilos de decisão, predominando em ambos os grupos o estilo comportamental, prevalecendo os aspectos interpessoais. Com isso, os agricultores demonstram habilidade para trabalhar bem em grupo, além de serem receptivos às sugestões dos demais, tentando evitar conflitos e buscar aceitação (ROWE; BOULGARIDES, 1983; ROWE; MASON; DILCKEL, 1998). Assim, as ações relacionadas com as normas subjetivas e as crenças de controle sugeridas anteriormente apresentam perspectivas promissoras, uma vez que os agricultores se demonstram receptivos a novas ideias.

Os resultados aqui apresentados sugerem que políticas públicas que enfatizam somente os aspectos econômicos são insuficientes para impulsionar a diversificação da produção agrícola. Talvez, os resultados desta pesquisa possam ser uma resposta para a incapacidade de as políticas públicas incentivarem a diversificação produtiva. Constata-se, portanto, que são necessárias políticas e ações que promovam desafios, realização e crescimento pessoal dos agricultores, com sentimento de orgulho em ser proprietário rural. Tais políticas devem ainda ser capazes de despertar nos agricultores valores como prazer nas tarefas da atividade rural, valor à saúde e vida no campo, além de evidenciar a liberdade de supervisionar suas próprias tarefas.

A incorporação dos estilos de decisão ao modelo da TCP demonstrou-se relevante por discutir conjuntamente aspectos até então pouco debatidos na literatura, uma vez que buscas preliminares, nos portais Web of Science e Science Direct, não identificaram estudos utilizando a TCP com a aplicação do modelo DSI ao público agrícola. Assim, pre- 
tendeu-se aqui fazer uma análise exploratória com tais variáveis, a fim de abrir caminhos para estudos futuros, os quais são extremamente necessários para dar continuidade à investigação do papel das construções psicológicas na formulação de estratégias de desenvolvimento no meio rural.

\section{REFERÊNCIAS}

ABDULAI, A.; CROLEREES, A. Determinants of income diversification amongst rural households in Southern Mali. Food Policy, 26, p. 437-452, 2001.

AJZEN, I. The theory of planned behavior. Organizational Behavior and Human Decision Processes, Amsterdan, 50, p. 179-211, 1991.

AJZEN, I. Attitudes, personality and behavior. 2. ed. Maidenhead: Open University Press, 2005.

AJZEN, I.; FISHBEIN, M. Understanding Attitudes and Predicting Social Behaviour. Englewood Cliffs, NJ: Prentice-Hall, 1980.

AMAZT, I. H.; IDRIS, A. R. Lecturers' Satisfaction towards University Management e Decision-making Styles in some Malaysian Public Universities. Procedia Social and Behavioral Sciences, 15, p. 3.957-3.970, 2011.

ARMSTRONG, S. J. The impact of supervisors' cognitive styles on the quality of research supervision in management education. British Journal of Educational Psychology, 74, p. 599-616, 2004.

BARBIERI, C.; MAHONEY, E. Why is diversification an attractive farm adjustment strategy? Insights from Texas farmers and ranchers. Journal of Rural Studies, 25, p. 58-66, 2009.

BARBIERI, C.; MSHENGA, P. The role of firm and owner characteristics on the performance of agritourism farms. Sociologia Ruralis, 48, p. 166-183, 2008.

BEEDELL, J.; REHMAN, T. Using social-psychology models to understand farmers'conservation behavior. Journal of Rural Studies, 16, p. 117-127, 2000.

BENJAMIN, C.; KIMHI, A. Farm work, off-farm work, and hired farm labour: estimating a discrete-choice model of French farm couples' labour decisions. European Review of Agricultural Economics, 33, p. 149$171,2006$.

BERGEVOET, R. H. M.; ONDERSTEIJN, C. J. M.; SAATKAM, H. W.; WOERKUM, C. M. J. Van; HUIRNE, R. B. M. Entrepreneurial behaviour of dutch dairy armers under a milk quota system: goals, objectives and attitudes. Agricultural Systems, 80, p. 1-21, 2004.

BORGES, J. A. R.; LANSINK, A. G. J. M. O. Comparing groups of Brazilian cattle farmers with different levels of intention to use improved natural grassland. Livestock Science, 178, p. 296-305, 2015.

BORGES, J. A. R.; LANSINK, A. G. J. M. O.; RIBEIRO, C. M.; LUTKE, V. Understanding farmers' intention to adopt improved natural grassland using the Theory of Planned Behavior. Livestock Science, 169, p. 163174, 2014

BRASIL. Lei № 8.629, de 25 de fevereiro de 1993. Dispõe sobre a regulamentação dos dispositivos constitucionais relativos à reforma agrária, previstos no Capítulo III, Título VII, da Constituição Federal. Presidência da República - Casa Civil. Brasília, DF, 25 fev. 1993. Disponível em: http://www.planalto.gov.br/ ccivil_03/LEIS/L8629.htm. Acesso em: 20 abr. 2014.

BURTON, R. J. F. Reconceptualising the "behavioural approach" in agricultural studies: a socio-psychological perspective. Journal of Rural Studies, 20, p. 359-371, 2004.

CEPEA. Centro de Estudos Avançados em Economia Aplicada. Escola Superior de Agricultura Luiz de Queiroz - Universidade de São Paulo (Esalq/USP). Preços ao produtor - valores nominais do leite (R\$̧/litro). São Paulo. Disponível em: http://cepea.esalq.usp.br/leite/?page=155. Acesso em: 4 abr. 2015.

CONNOR, P. E.; BECKER, B.W. Personal value systems and decision-making styles of public managers. Public Personnel Management, 32, p. 155-180, 2003.

DALCIN, D. Os estilos de tomada de decisão e o desempenho econômico das propriedades rurais de Palmeira das Missões/RS. 2013. Tese (Doutorado em Agronegócios) - Universidade Federal do Rio Grande do Sul, Centro de Estudos e Pesquisas em Agronegócios, Porto Alegre, Brasil, 2013.

DUTRA, A. da S. O processo decisório de implantação de estrutura para armazenagem de soja ao nível de propriedade rural na região de Santo Ângelo/RS. 2008. Dissertação (Mestrado em Agronegócios) - Centro de Estudos e Pesquisas em Agronegócios, Universidade Federal do Rio Grande do Sul, Porto Alegre, Brasil, 2008.

ELLIOTT, J.; SNEDDON, J.; LEE, J. A.; BLACHE, D. Producers have a positive attitude toward improving lamb survival rates but may be influenced by enterprise factors and perceptions of control. Livestock Science, 140, p. 103-110, 2011. 
ELLIS, F. Rural livelihoods and diversity in developing countries. Oxford: Oxford University, 2000a.

ELLIS, F. The determinants of rural livelihood diversification in developing countries. Journal of Agricultural Economics, 51, p. 289-302, 2000b.

FERGUSON, R.; HANSSON, H. Measuring Embeddedness and Its Effect on New Venture Creation - A Study of Farm Diversification. Managerial and Decision Economics, 36, p. 314-325, 2015.

FERGUSON, R.; HANSSON, H. Expand or exit? Strategic decisions in milk production. Livestock Science, 155, p. 415-423, 2013.

FIELDING, K. S.; TERRY, D. J.; MASSER, B. M.; BORDIA, P.; HOGG, M. A. Explaining landholders' decisions about riparian zone management: The role of behavioural, normative, and control beliefs. Journal of Environmental Management, 77, p. 12-21, 2005.

FIELDING, K. S.; TERRY, D. J.; MASSER, B. M.; HOGG, M. A. Integrating social identity theory and the theory of planned behaviour to explain decisions to engage in sustainable agricultural practices. British Journal of Social Psychology, 47, p. 23-48, 2008.

FOX, T. L.; SPENCE, J. W. An examination of the decision styles of project managers: Evidence of significant diversity. Information \& Management, 36, p. 313-320, 1999.

GASSON, R. Goals and values of Farmers. Journal of Agricultural and Resource Economics, 24, p. 521-537, 1973.

GASSON, R.; POTTER, C. Conservation Through Land Diversion: a Survey of Farmers Attitudes. Journal of Agricultural Economics, Reading 39, p. 340-351, 1988.

HAIR, J. F.; BLACK, W. C.; BABIN, B. J.; ANDERSON, R. E. Multivariate Data Analysis, 7. ed. Prentice Hall, New Jersey, 2010.

HANSSON, H.; FERGUSON, R. Factors influencing the strategic decision to further develop dairy production: A study of farmers in central Sweden. Livestock Science, 135, p. 110-123, 2011.

HANSSON, H.; FERGUSON, R.; OLOFSSON, C. Understanding the diversification and specialization of farm businesses. Agricultural and Food Science, 19, p. 269-283, 2010.

HANSSON, H.; FERGUSON, R.; OLOFSSON, C. Psychological Constructs Underlying Farmers' Decisions to Diversify or Specialise their Businesses - An Application of Theory of Planned Behaviour. Journal of Agricultural Economics, 63, p. 465-482, 2012.

HANSSON, H.; FERGUSON, R.; OLOFSSON, C.; RANTAMÄKI-LAHTINEN, L. Farmers? motives for diversifying their farm business: the influence of family. Journal of Rural Studies, 32, p. 240-250, 2013.

HJALAGER, A. M. Agricultural diversification into tourism: evidence of a European Community Development Programme. Tourism Manage, 17, p. 103-111, 1996.

hOFFMANN, R.; SERRANO, O.; NEVES, E. M.; THAME, A. C. DE M.; ENGLER J. J. de C. Administração da empresa agrícola. 7. ed. São Paulo: Pioneira, 1987.

ILBERY, B. W. Farm diversification as an adjustment strategy on the urban fringe of the West Midlands. Journal of Rural Studies, 7, p. 207-218, 1991.

JACOBY, J. M. Relationship between principals' decision making styles and technology acceptance and use. 2006. Doctoral Thesis, University of Pittsburgh, 2006.

JAMIAN, L. S.; SIDHU, G. K.; APERAPAR, P. S. Managerial Decision Styles of Deans in Institutions of Higher Learning. Procedia - Social and Behavioral Sciences, 90, p. 278-287, 2013.

JONGENEEL, R. A.; POLMAN, N. B.; SLANGEN, L. H. Why are Dutch farmers going multifunctional? Land Use Policy, 25, p. 81-94, 2008.

LANGE, A.; PIORR, A.; SIEBERT, R.; ZASADA, I. Spatial differentiation of farm diversification: how rural attractiveness and vicinity to cities determine farm households' response to the CAP. Land Use Policy, 31, p. 136-144, 2013.

LÄPPLE, D.; KELLEY, H. Understanding the uptake of organic farming: Accounting for heterogeneities among Irish farmers. Ecological Economics, 88, p. 11-19, 2013.

LAUWERE, C.; ASSELDONK, M.; RIET, J.; HOOP, J.; PIERICK, E. Understanding farmers' decisions with regard to animal welfare: The case of changing to group housing for pregnant sows. Livestock Science, 143, p. 151-161, 2012.

MACHADO, J. A. D.; OLIVEIRA, L. M. de; SCHNORRENBERGER, A. Compreendendo a tomada de decisão do produtor rural. Fortaleza: Sociedade Brasileira de Economia e Sociologia Rural (Sober), 2006.

MALHOTRA, N. K. Marketing Research: An Applied Orientation. 6. ed. Nova Jersey, EUA: Prentice Hall, 897, 2009. 
MARTÍNEZ-GARCÍA, C. G.; DORWARD, P.; REHMAN, T. Factors influencing adoption of improved grassland management by small-scale dairy farmers in central Mexico and the implications for future research on smallholder adoption in developing countries. Livestock Science, 152, p. 228-238, 2013.

MCNALLY, S. Farm diversification in England and Wales: what can we learn from the farm business survey? Journal of Rural Studies, 17, p. 247-257, 2001.

MERANER, M.; HEIJMAN, W.; KUHLMANC, T.; FINGER, R. Determinants of farm diversification in the Netherlands. Land Use Policy, 42, p. 767-780, 2015.

MISHRA, A. K.; EL-OSTA, H. S.; SANDRETTO, C. L. Factors affecting farm Enterprise diversification. Agricultural Finance Review, 64, p. 151-166, 2004.

NILSSON, P. A. Staying on farms: an ideological background. Annals of Tourism Research, 29, p. 7-24, 2002. NORDER, L. A. C. Mercantilização da agricultura e desenvolvimento territorial. In: SCHNEIDER, S. (org.) A diversidade da agricultura familiar. 2. ed. Porto Alegre: Editora da UFRGS, 2009.

PARKER, A. M.; FISCHHOFF, B. Decision-making Competence: External Validation through an Individual-differences Approach. Journal of Behavioral Decision Making, 18, p. 1-27, 2005.

PFEIFER, C.; JONGENEEL, R. A.; SONNEVELD, M. P.; STOORVOGEL, J. J. Landscape properties as drivers for farm diversification: a Dutch case study. Land Use Policy, 26, p. 1.106-1.115, 2009.

PLOEG, J. D. Van der; ROEP, D. Multifunctionality and rural development the actual situation in Europe. In: HUYLENBROECK, G. Van; DURAND, G. (ed.). Multifunctional Agriculture. A New Paradigm for European Agriculture and Rural Development. Hampshire, England: Ashgate; Aldershot, 2003. p. 37-54.

ROBBINS, S. P. Comportamento organizacional. 11. ed. São Paulo: Pearson Prentice Hall, 2006.

ROWE, A. J.; BOULGARIDES, J. D. Decision Styles: A Perspective. Leadership e Organization. Development Journal, 4, p. 3-9, 1983.

ROWE, A. J.; BOULGARIDES, J. D. Managerial Decision Making. New York: Macmillan Publishing Company, 1992.

ROWE, A. J.; MASON, R. O. Managing with style: A guide to understand, assessing, and improving decision making. San Francisco: Jossey-Bass Publisher, 1987.

ROWE, A. J.; MASON, R. O.; DILCKEL, K. E. Strategic Management. A Methodological Approach. Massachusetts: Addison-Wesley Publishing Company, 1998.

SCHNEIDER, S. A pluriatividade na agricultura familiar. 2. ed. Porto Alegre: Editora da UFRGS, 2009.

TURNER, M.; WHITEHEAD, D.; BARR, D.; FOGERTY, M.; ERRINGTON, A.; LOBLEY, M.; REED, M. Farm Diversification Activities: Benchmarking Study 2002 - Final Report to Defra, 2003. (Exeter: University of Exeter and University of Plymouth).

VIK, J.; MCELWEE, G. Diversification and the entrepreneurial motivations of farmers in Norway. Journal of Small Business Management, 49, p. 390-410, 2011.

YAZDANPANAH, M.; HAYATI, D.; HOCHRAINER-STIGLER, S.; ZAMANI, G. H. Understanding farmers' intention and behavior regarding water conservation in the Middle-East and North Africa: A case study in Iran. Journal of Environmental Management, 135, p. 63-72, 2014. 